\title{
Numerical estimation of lunar X-ray emission for X-ray spectrometer onboard SELENE
}

\author{
Kazunori Ogawa $^{1,2}$, Tatsuaki Okada ${ }^{1}$, Kei Shirai $^{1}$, and Manabu Kato ${ }^{1}$ \\ ${ }^{1}$ Department of Planetary Science, Instutute of Space and Astronautical Science, Japan Aerospace Exploration Agency, \\ 3-1-1 Yoshinodai, Sagamihara, Kanagawa 229-8510, Japan \\ ${ }^{2}$ Department of Earth and Planetary Sciences, Tokyo Institute of Technology, \\ 2-12-1 Ookayama, Meguro-ku, Tokyo 152-8550, Japan
}

(Received April 21, 2007; Revised September 10, 2007; Accepted October 22, 2007; Online published April 9, 2008)

\begin{abstract}
We conducted a numerical estimation of lunar X-ray spectra, which is applicable for lunar X-ray fluorescence observations using an X-ray spectrometer (XRS) onboard the SELENE orbiter, with an improved simulation model. We investigated the integration times of measurements for six elements $(\mathrm{Mg}, \mathrm{Al}, \mathrm{Si}, \mathrm{Ca}, \mathrm{Ti}$, and $\mathrm{Fe})$ to achieve signal-to-background ratio of over 10 under various solar conditions. The results of these calculations indicate that expected along-the-track spatial resolutions of a single orbital path for $\mathrm{Mg}, \mathrm{Al}$ and $\mathrm{Si}$ will be $<90 \mathrm{~km}$ and $20 \mathrm{~km}$ under normal and active Sun conditions, respectively. $\mathrm{Ca}$, Ti and $\mathrm{Fe}$ will be also detectable with a spatial resolution of $20 \mathrm{~km}$ during the periods active solar flares over M1 class happen to occur.
\end{abstract}

Key words: Lunar X-ray, numerical simulation, X-ray spectrometer, XRS, SELENE.

\section{Introduction}

An X-ray fluorescence spectrometer (XRS) has been developed for the SELENE (SELenological and ENgineering Explorer) mission, a Japanese lunar polar orbiter mission that will be launched in the summer of 2007, with the aim of globally mapping major elemental composition. Remote $\mathrm{X}$-ray fluorescence spectrometry during spacecraft orbits is an available method to determine the composition of chemical elements on the surface of atmosphere-free rocky planets. Electrons in atoms composing the uppermost planetary surface of less than $1 \mathrm{~mm}$ deep are excited by the irradiation of solar X-rays, and generate characteristic X-rays to space while moving back toward the ground state immediately. The energies of these fluorescent X-ray lines are a direct indication of the elemental composition of the surface layer.

Detailed map of elemental composition of the lunar surface is an important key for an understanding of the lunar chemical and geological history, and the origin of the Moon. The pioneering work of measuring lunar fluorescent X-rays was made on the Luna 12 orbiter, which acquired positive indications that the Sun produces measurable fluorescent Xrays, and on Apollo 15 and 16, which successfully detected fluorescent X-rays of $\mathrm{Mg}, \mathrm{Al}$ and $\mathrm{Si}$ from approximately 9\% of the lunar surface (Adler et al., 1972a, b, 1973a, b; Adler and Trombka, 1977). The recent approach was made by the D-CIXS (Demonstration of a Compact Imaging Xray Spectrometer) on SMART-1 orbiter, which measured Xray lines including calcium by improved detectors (Grande et al., 2003, 2007). At present, X-ray spectrometry of the Moon is being newly attempted by some lunar missions,

Copyright (c) The Society of Geomagnetism and Earth, Planetary and Space Sciences (SGEPSS); The Seismological Society of Japan; The Volcanological Society of Japan; The Geodetic Society of Japan; The Japanese Society for Planetary Sciences; TERRAPUB. one of which is SELENE (Okada et al., 2002; Kato et al., 2007).

Quantitative analyses of the lunar X-ray spectra for future lunar X-ray missions have to date been conducted by numerical X-ray interaction models (e.g., Okada, 1996; Okada et al., 2002; Clark and Trombka, 1997; Clark, 1997). For the XRS onboard SELENE, the estimation must be recalculated with its improved instrumental performance and orbital configuration in order to optimize operational plans and exact data analyses during flight. We performed the computations of the prospective lunar X-ray spectra measured by detectors of the XRS by a model with the anomalous X-ray scattering collection of atomic scattering factor and the Doppler broadening effect of incoherent X-ray scattering. With respect to the solar X-rays as excitation source of lunar X-rays, line components in the spectra and their scatterings were also taken into account. The lunar surface was assumed to be flat and homogeneous. The surface particle size (Kuwada et al., 1997; Maruyama et al., 2007, 2008) and the irregular topographical features, which have some level of influence on the intensity of the lunar X-rays, are also important and must also be considered for detailed analyses, but they are not considered here.

\section{X-Ray Spectrometer Onboard SELENE}

The XRS onboard SELENE is a charge-coupled device (CCD) based energy dispersive X-ray spectrometer. It was designed for global mapping of major rock-forming elements on the lunar surface, targeting $\mathrm{Mg}, \mathrm{Al}$ and Si mainly, and $\mathrm{Ca}, \mathrm{Ti}$ and $\mathrm{Fe}$ secondarily, within $10 \%$ error of elemental ratios (Okada et al., 2002). The XRS system consists of three components, the XRF-A as the main sensor, SOL-B as a direct solar X-ray monitor and SOL-C as a standard sample monitor. The XRF-A carries out measurements of 
Table 1. References to the fundamental parameters.

\begin{tabular}{lll}
\hline Definition & Term & Reference \\
\hline Mass absorption coefficient & $\mu$ & Chantler et al. (2005) \\
X-ray absorption ratio of electron orbits & $R$ & Calculated from jump ratio in mass absorption \\
& & coefficient data tables (Chantler et al., 2005). \\
Fluorescence yield & $\omega$ & Hubbell et al. (1994) \\
Intensity ratio of emission lines & $P$ & Salem et al. (1974) \\
Normal atomic scattering factor & $f_{0}$ & Cromer and Waber (1974) \\
Atomic scattering factor correction terms & $f_{n(\neq 0)}$ & Chantler et al. (2005) \\
Incoherent scattering function & $S$ & Calculated by the method in Brusa et al. (1996). \\
Fluorescence energy & & Bearden (1967) \\
Absorption edge energy & & Chantler et al. (2005) \\
\hline
\end{tabular}

the lunar X-rays with $16 \mathrm{CCDs}$, which provide a total effective area of $100 \mathrm{~cm}^{2}$. The CCDs are sensitive to soft X-rays at $0.7-10 \mathrm{keV}$, which includes fluorescent $\mathrm{K}$ lines of all the target elements. A typical size of unit energy channel of the detectors is approximately $10 \mathrm{eV}$, and the energy resolution was estimated to be $<160 \mathrm{eV}$ at $\mathrm{Si}-\mathrm{K} \alpha(1.74 \mathrm{keV})$ and $<200 \mathrm{eV}$ at $\mathrm{Fe}-\mathrm{K} \alpha(6.40 \mathrm{keV})$ as the full width half maximum (FWHM) of the line peaks. X-ray collimators in front of the CCDs adjust their field-of-view to $12^{\circ} \times 12^{\circ}$, and the footprint to $20 \times 20 \mathrm{~km}^{2}$ on the lunar surface when observed from a 100-km altitude orbit. The SOL-B measures direct solar X-rays at 1-20 keV using two Si-PIN detectors with hemispherical wide view and energy resolution of $<500 \mathrm{eV}$ at $\mathrm{Fe}-\mathrm{K} \alpha$ as the FWHM. The SOL-C performs as a calibrator for XRF-A data with a single CCD, measuring X-rays emitted from the standard sample exposed to the Sun. The standard sample is a glassy plate whose composition is similar to the lunar rock average. The intensity of the lunar fluorescent lines strongly depends on the solar spectrum (e.g., Adler et al., 1972a; Clark and Trombka, 1997). The SOL-B and SOL-C will correct any spectral fluctuation caused by various solar activity. Detailed instrumentation and performances of the XRS are reported in other papers (Yamamoto et al., 2007; Shirai et al., 2008).

SELENE will observe the Moon from nearly polar 100$\mathrm{km}$ circular orbits at a ground speed of approximately $1.5 \mathrm{~km} / \mathrm{s}$ (Kato et al., 2007). The XRS will operate continuously, providing 20-km swath lunar compositional measurements about half of the time that the surface is illuminated. The quality of the X-ray fluorescence measurements is correlated with the higher solar incidence angles of the lower latitudes. Such data require shorter integration times. On the other hand, measurements made with the lower solar incidence angle require longer integration times because of the weak irradiation of the Sun. This discrepancy is somewhat compensated for by the far greater overlap between successive orbits that occurs in polar regions. In fact, SELENE XRS will provide coverage of the entire lunar surface, including the equatorial regions, 6 times over.

\section{Numerical Model for Lunar X-Ray Emission}

The numerical model for the lunar X-ray spectra is described in this section. X-rays from the lunar surface are generated predominantly by interactions in the surface material with solar X-rays, while other photons and energetic particles contribute only negligibly $\left(<10^{-4}\right.$, e.g., Hayakawa and Matsuoka, 1962). Therefore, lunar X-ray spectra are numerically explained by solar X-ray spectra and their interactions on the Moon. In this study, lunar X-ray spectra were computed by a fast simulation technique of X-ray interactions and spectrum models of solar X-rays similar to an earlier study by Okada (1996). References to the fundamental parameters used in the following calculations are shown in Table 1.

\subsection{X-ray interaction model}

Three different solar-induced processes, including fluorescence and coherent and incoherent scatter, generate $\mathrm{X}$ rays in the lunar regolith to a depth of less than $1 \mathrm{~mm}$. The following equation, the so-called Shiraiwa-Fujino equation, explains the relationship between the primary X-ray irradiation and the subsequent X-ray fluorescence generation, assuming that the sample has a flat surface, homogeneous interior without structure and elemental concentration and infinite depth (Shiraiwa and Fujino, 1966). The equation is also applicable to the X-ray scattering (Okada, 1996). X-ray intensity $I$ and energy $E^{\prime}$ produced in element $i$ by primary $\mathrm{X}$-ray flux $J$ as a function of energy $E$ is expressed as

$$
I\left(E^{\prime}\right)=\int_{E=0}^{\infty} \frac{w_{i} \xi_{i} J(E)}{\mu(E)+\mu\left(E^{\prime}\right) \frac{\cos \theta}{\cos \phi}} \mathrm{d} \Omega \mathrm{d} E,
$$

where $w_{i}$ is the mass fraction of $i$ th element, $\mu(E)$ is the total X-ray mass absorption coefficient (or mass attenuation coefficient) of the sample at energy $E, \theta$ is the primary $\mathrm{X}$ ray incident angle to normal of the sample surface, $\phi$ is the $\mathrm{X}$-ray emission angle to normal, $\Omega$ is the detectable solid angle of X-ray generation and $\xi_{i}$ is a reaction factor of $i$ th element, that is the mass absorption coefficient per detection solid angle for each interaction of the target element. The formula is integrated over an energy range of $E$ if the primary X-rays are not monochromatic. The total mass absorption coefficient is calculated from that of each component element $\mu_{i}$ as follows,

$$
\mu=\sum_{i} w_{i} \mu_{i}
$$

Each of the three processes, X-ray fluorescence and coherent, and incoherent X-ray scattering can be described with the formulation of Eq. (1) with different $\xi_{i}$ for different primary interactions in the slab. Total errors, including un- 
certainties of published fundamental parameters, were estimated to be less than $10 \%$ of both fluorescent and scattering $\mathrm{X}$-ray photon counts.

3.1.1 X-ray fluorescence $X$-ray fluorescence is generally understood by the following process: a photoelectric absorption of a primary X-ray photon by an atom, followed by the transfer of an electron in the outer shell orbit down to the vacancy, resulting in a photon generation whose energy equals the potential difference between the two energy levels. $\xi_{i}$ for this process is a product of the mass absorption coefficient for the photoelectric absorption and the probability of X-ray emission for a vacancy.

In the case that an electron in orbital $k$ of an atom of $i$ th element traps the primary $\mathrm{X}$-ray photon and escapes from the atom and an electron in orbital $l$ falls down to the vacancy, $\xi_{i}$ is written as

$$
\xi_{i}=\mu_{(\mathrm{pe}) i}(E) R_{i k} \omega_{i k} P_{i k l} \frac{1}{4 \pi},
$$

where, $\mu_{(\mathrm{pe}) i}(E)$ is the photoelectric mass absorption coefficient of $i$ th element at energy $E, R_{i k}$ is the probability of trapping the primary photons in $k$ th orbit, $\omega_{i k}$ is the fluorescence yield for orbital $k$, meaning the probability that X-ray fluorescence generation occurs from the vacancy, and $P_{i k l}$ is the probability of an electron in the orbital $l$ falling down to the vacancy. The X-ray fluorescence generation is statistically isotropic so that the formula is independent of the emission angle and is divided by $4 \pi$ for yield per unit solid angle. $E^{\prime}$ is determined uniquely by the above $i, k$ and $l$ as the fluorescent line energy. In addition, the Coster-Kronig hole transfer probability must be considered in Eq. (3) for $\mathrm{L}$ or higher shell vacancies. However, $\mathrm{L}$ or higher lines are not mentioned here because these lines of the lunar major elements are out of the detectable energy range of the XRS.

3.1.2 X-ray scattering Photons in the energy range 0.7-10 keV scatter in following two ways, coherent (Rayleigh) and incoherent (Compton) scattering, mostly by electrons in the material. These two components cause the background continuum for the fluorescent line emission. It is generally known that the intensity of those scatterings depends on the scattering angle and the photon energy, and it is the weakest if scattered to $\pi / 2$ radian from primary direction. The efficiency factors $\xi_{i}$ of the scatterings are derived from the following differential cross section (DCS) per atom,

$$
\xi_{i}=\frac{\mathrm{d} \mu_{(\mathrm{coh} / \mathrm{inc}) i}}{\mathrm{~d} \Omega}=\frac{N}{A_{i}} \frac{\mathrm{d} \sigma_{(\mathrm{coh} / \mathrm{inc}) i}}{\mathrm{~d} \Omega},
$$

where $N$ is Avogadro's constant, $A_{i}$ is the atomic mass of $i$ th element and $\sigma_{i}$ is the cross section of each scattering for a single atom of $i$ th element.

DCS of the coherent scattering for an unpolarized photon is given by

$$
\frac{\mathrm{d} \sigma_{(\mathrm{coh}) i}}{\mathrm{~d} \Omega}=\frac{r_{e}^{2}}{2}\left(1+\cos ^{2} \alpha\right) f_{i}^{2},
$$

where $r_{e}$ is the classical electron radius, $\alpha$ is the scattering angle ( $\pi$ minus the angle between primary incidence and scattered directions) and $f_{i}$ is the atomic scattering factor of the $i$ th element, which depends on the scattering angle and the primary photon energy. The energy of coherently scattered photon is equal to the primary, i.e. $E^{\prime}=E$.

Previous studies explained that the atomic scattering factor, or atomic form factor, $f$ for the atom of the atomic number $Z$ is given by following components (e.g., Chantler, 2000),

$$
\begin{aligned}
& \operatorname{Re}(f)=f_{0}+f_{1}+f_{\text {rel }}-Z+f_{\mathrm{NT}}, \\
& \operatorname{Im}(f)=f_{2},
\end{aligned}
$$

where $f_{0}$ is the conventional (normal) atomic scattering factor, $f_{1}$ is the non-relativistic anomalous dispersion correction, $f_{\text {rel }}$ is the relativistic correction, $f_{\mathrm{NT}}$ is the nuclear Thomson scattering correction and $f_{2}$ corrects anomalous decrease of the coherent scattering near energies of the photo-absorption edges. Compared to the simplification $f=f_{0}$ for fast estimates, the corrections reduce approximately $10 \%$ of the scattering counts mainly in $1-2 \mathrm{keV}$ range in the present case due to the anomalous scatterings near the edges.

The flux of incoherent scattering photons is small relative to coherent scattering in this energy range, and its fraction is approximately $10 \%$ at maximum when the scattering angle is around $\pi / 2$ radian. DCS of the incoherent scattering for an unpolarized photon is given (Ribberfors and Berggren, 1982) as

$$
\begin{aligned}
\frac{\mathrm{d} \sigma_{(\mathrm{inc}) i}}{\mathrm{~d} \Omega} & =\frac{\mathrm{d} \sigma_{(\mathrm{KN})}}{\mathrm{d} \Omega} S_{i}(E, \alpha), \\
\frac{\mathrm{d} \sigma_{(\mathrm{KN})}}{\mathrm{d} \Omega} & =\frac{r_{e}^{2}}{2}\left(\frac{E_{c}}{E}\right)^{2}\left(\frac{E_{c}}{E}+\frac{E}{E_{c}}-\sin ^{2} \alpha\right),
\end{aligned}
$$

where $\sigma_{(\mathrm{KN})}$ is the Klein-Nishina cross section, $E_{c}$ is the Compton energy, and $S_{i}$ is the incoherent scattering function. It is generally known that the energy of the incoherently scattered photons is reduced to $E_{c}$,

$$
E_{c}=E^{\prime}=\frac{m_{e} c^{2} E}{m_{e} c^{2}+E(1-\cos \alpha)},
$$

where $m_{e}$ is the electron mass, and $c$ is the velocity of light. For a more appropriate description of the incoherent spectra, energy of the incoherently scattered photons is broadened and has a distribution with a central focus on $E_{c}$, which effect is called Doppler broadening, due to the initial energy distribution of target electrons. Therefore, in this case, Eq. (8) is rewritten with the double differential cross section (DDCS) as

$$
\frac{\mathrm{d}^{2} \sigma_{(\text {inc }) i}}{\mathrm{~d} \Omega \mathrm{d} E^{\prime}}=\frac{\mathrm{d} \sigma_{(\mathrm{KN})}}{\mathrm{d} \Omega} S_{i}\left(E, E^{\prime}, \alpha\right) .
$$

The Doppler broadening effect is often considered to be insignificant for a case with continuum spectra without strong emission lines in the primary irradiation, but our model includes the effect to achieve more accurate estimates, thereby allowing a more realistic assessment of the implications. We use here a fast simulation algorithm suggested by Brusa et al. (1996) for the incoherent scattering calculations. The influence of the broadening effect was within $10 \%$ of the incoherent spectra without broadening, and within $1 \%$ taking into account all scattering effects. 
Table 2. Plasma temperatures and emission measures $\left(\mathrm{cm}^{-3}\right)$ of the model solar X-ray spectra.

\begin{tabular}{cccccc}
\hline GOES flare class & $4 \mathrm{MK}$ & $8 \mathrm{MK}$ & $12 \mathrm{MK}$ & $16 \mathrm{MK}$ & $20 \mathrm{MK}$ \\
\hline A3.0 & $2.8 \mathrm{e}+47$ & $1.0 \mathrm{e}+46$ & & & \\
B1.2 & $5.6 \mathrm{e}+47$ & $8.0 \mathrm{e}+46$ & $2.5 \mathrm{e}+46$ & & \\
C1.2 & $2.0 \mathrm{e}+48$ & $5.0 \mathrm{e}+47$ & $3.2 \mathrm{e}+47$ & $2.5 \mathrm{e}+47$ & \\
M1.3 & $4.0 \mathrm{e}+48$ & $2.5 \mathrm{e}+48$ & $2.5 \mathrm{e}+48$ & $2.5 \mathrm{e}+48$ & $2.5 \mathrm{e}+48$ \\
\hline
\end{tabular}

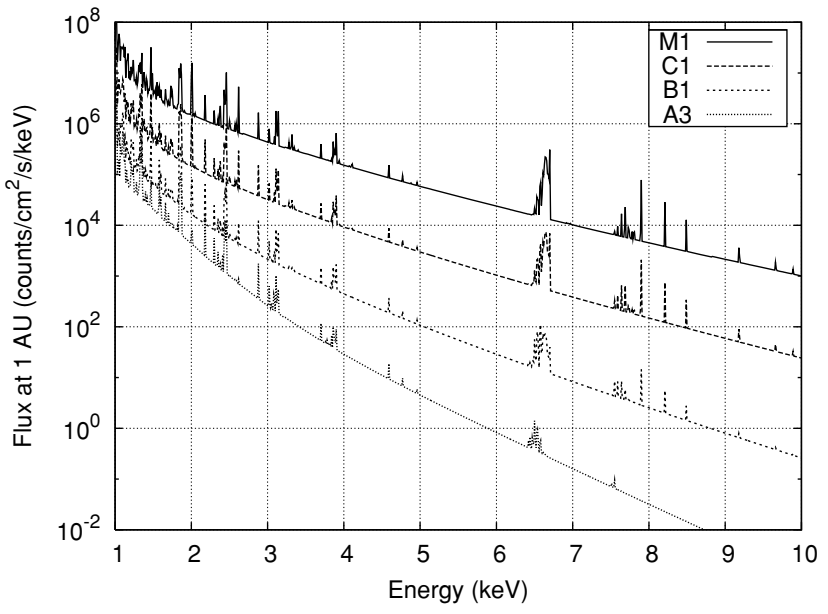

Fig. 1. Solar X-ray spectra models of each solar condition at 1 AU were calculated by the MEKAL model with the parameters in Table 2. A3 represents a typical solar spectrum during the XRS operation period, and B1, C1 and M1 represent spectra of each occasional flare state. The higher level flares occur less frequently. During the SELENE mission, a few tens of M1 flares are predicted to occur.

\subsection{Solar X-ray spectrum}

The spectral shape of the lunar X-rays significantly depends on the solar X-ray spectrum. The solar X-rays consist of a continuum as the result of the thermal bremsstrahlung and radiative recombination, and characteristic emission lines of solar corona. It is difficult to determine the solar $\mathrm{X}$-ray spectrum accurately because it is highly variable in time. At present, data from GOES satellites are available to estimate the solar X-ray flux. Solar flare classes are A, B, $\mathrm{C}, \mathrm{M}$ and $\mathrm{X}$, indicating the order of magnitude of the solar $\mathrm{X}$-ray flux in ascending order.

Solar X-ray spectral models at four representative solar activity levels of A3, B1, C1, and M1 were used in this study (Fig. 1). Each of these models is calculated by the summation of spectra having multiple plasma temperatures and the emission measures of the Mewe-Kaastra-Liedahl (MEKAL) collisional ionization equilibrium plasma model using SPEX software (SPEctral X-ray and UV modeling, analysis and fitting, http://www.sron.nl/divisions/hea/spex/index.html). Figure 1 shows the model solar spectra, and Table 2 shows parameters (plasma temperatures and emission measures) of the MEKAL calculations. These model spectra agree with the results of past studies (e.g., Clark and Trombka, 1997) in their continuum shape and flux, but our model includes the emission line component. In Fig. 1, A3.0 is assumed as a "typical" or most quiescent solar state during the XRS operations. $\mathrm{B}, \mathrm{C}$ and $\mathrm{M}$ represent occasional flare conditions, respectively. This assumption is a lower limit of the solar intensity. The typical condition of the Sun is able to be a little more active in 2007-2008 due to the 11-year solar cycle. During the actual XRS operations, the solar X-ray spectrum is simultaneously observed by the SOL-B for accurate conversions from the lunar fluorescence counts into the elemental abundances.

The solar strength on the lunar surface correlates with the distance from the Sun and the solar incidence angle. The solar X-ray strength $J$ per footprint of the XRS is written as

$$
J(E)=\frac{B \cos \theta}{D^{2}} J_{0}(E),
$$

where $B$ is the size of the footprint in $\mathrm{cm}^{2}, D$ is the distance from the Sun in $\mathrm{AU}$, and $J_{0}$ is the solar flux per $\mathrm{cm}^{2}$ at $1 \mathrm{AU}$ shown in Fig. 1. The primary flux given by Eq. (12) was used in the lunar X-ray model.

\section{Calculations and Results of Lunar X-Rays}

Computations of expected lunar X-ray spectra detected by the XRS onboard SELENE were carried out using the methods mentioned above. In this section, we show the configuration and some of the results of our simulations. SELENE orbits the Moon in polar circular orbits at an altitude of $100 \mathrm{~km}$, and the XRS is always turned on and observes lunar surface pointing exactly at nadir. As mentioned in Section 2, the XRS has total detection area of $100 \mathrm{~cm}^{2}$ and the square footprint of $20 \times 20 \mathrm{~km}^{2}$ area, yielding the highest X-ray collection efficiency at the center of the footprint.

In terms of the simulation parameters, X-ray emission angle $\phi$ and detection solid angle $\Omega$ are constant during the mission, $\phi=0$, and $\Omega \sim 10^{-12}$, respectively. Primary incidence angle $\theta$ varies in $0 \leq \theta<\pi / 2$ periodically with the solar altitude (lunar latitude and $\beta$ angle of orbit) at the time. Four solar spectra at solar activities A3, B1, C1, and M1 given by Eq. (12) were used as the primary excitation source.

For the elemental composition of the virtual lunar surface, average compositions of two Apollo landing sites, 12 and 16, were used as being representative of soils of lunar maria and highlands. Additionally, six typical rocks from the Apollo missions were added to the simulations: high-Ti mare basalt 70215, aluminous low-Ti mare basalt 14053, KREEP basalt 15382, ferroan anorthosite 15415 as a symbolic rock of highlands, and norite 77215 and troctolite 76535 representing the $\mathrm{Mg}$-suite. Detailed compositions of major elements of these samples are shown in Table 3.

In the XRS, the X-ray detection efficiency is determined by beryllium filters in front of the CCDs and the effective and ineffective layer thicknesses of the CCDs. Total detection efficiency at each photon energy was applied to the 
Table 3. Compositions (wt\%) of lunar samples for simulations (McKay et al., 1991; Taylor et al., 1991).

\begin{tabular}{|c|c|c|c|c|c|c|c|c|}
\hline & $\begin{array}{c}\text { Apollo } 12 \\
\text { site } \\
\text { average }\end{array}$ & $\begin{array}{c}\text { Apollo } 16 \\
\text { site } \\
\text { average }\end{array}$ & $\begin{array}{c}\text { High-Ti } \\
\text { basalt } \\
70215\end{array}$ & $\begin{array}{c}\text { High-Al } \\
\text { basalt } \\
14053\end{array}$ & $\begin{array}{c}\text { KREEP } \\
\text { basalt } \\
15382\end{array}$ & $\begin{array}{c}\text { Ferroan } \\
\text { anorthosite } \\
15415\end{array}$ & $\begin{array}{l}\text { Norite } \\
77215\end{array}$ & Troctolite \\
\hline $\mathrm{Na}_{2} \mathrm{O}$ & 0.54 & 0.46 & 0.36 & - & 0.87 & 0.36 & 0.40 & 0.23 \\
\hline $\mathrm{MgO}$ & 9.3 & 5.7 & 8.4 & 8.5 & 7.83 & 0.26 & 12.5 & 20.0 \\
\hline $\mathrm{Al}_{2} \mathrm{O}_{3}$ & 12.9 & 27.3 & 8.8 & 13.6 & 16.9 & 35.6 & 15.0 & 19.9 \\
\hline $\mathrm{SiO}_{2}$ & 46.3 & 45.0 & 37.8 & 46.4 & 52.5 & 44.5 & 51.5 & 43.0 \\
\hline $\mathrm{P}_{2} \mathrm{O}_{3}$ & 0.4 & 0.11 & - & - & 0.43 & - & 0.085 & 0.018 \\
\hline $\mathrm{S}$ & - & 0.07 & - & - & - & - & - & - \\
\hline $\mathrm{K}_{2} \mathrm{O}$ & 0.31 & 0.17 & 0.05 & 0.10 & 0.53 & 0.015 & 0.173 & 0.028 \\
\hline $\mathrm{CaO}$ & 10.7 & 15.7 & 10.7 & 11.2 & 9.43 & 20.4 & 9.1 & 10.8 \\
\hline $\mathrm{TiO}_{2}$ & 3.0 & 0.54 & 13.0 & 2.6 & 1.90 & 0.02 & 0.33 & 0.05 \\
\hline $\mathrm{Cr}_{2} \mathrm{O}_{3}$ & 0.34 & 0.33 & 0.41 & - & 0.26 & 0.003 & 0.36 & 0.11 \\
\hline $\mathrm{MnO}$ & 0.22 & 0.22 & 0.27 & 0.26 & 0.12 & 0.006 & 0.16 & 0.065 \\
\hline $\mathrm{FeO}$ & 15.1 & 5.4 & 19.7 & 16.8 & 9.02 & 0.21 & 9.9 & 5.0 \\
\hline
\end{tabular}
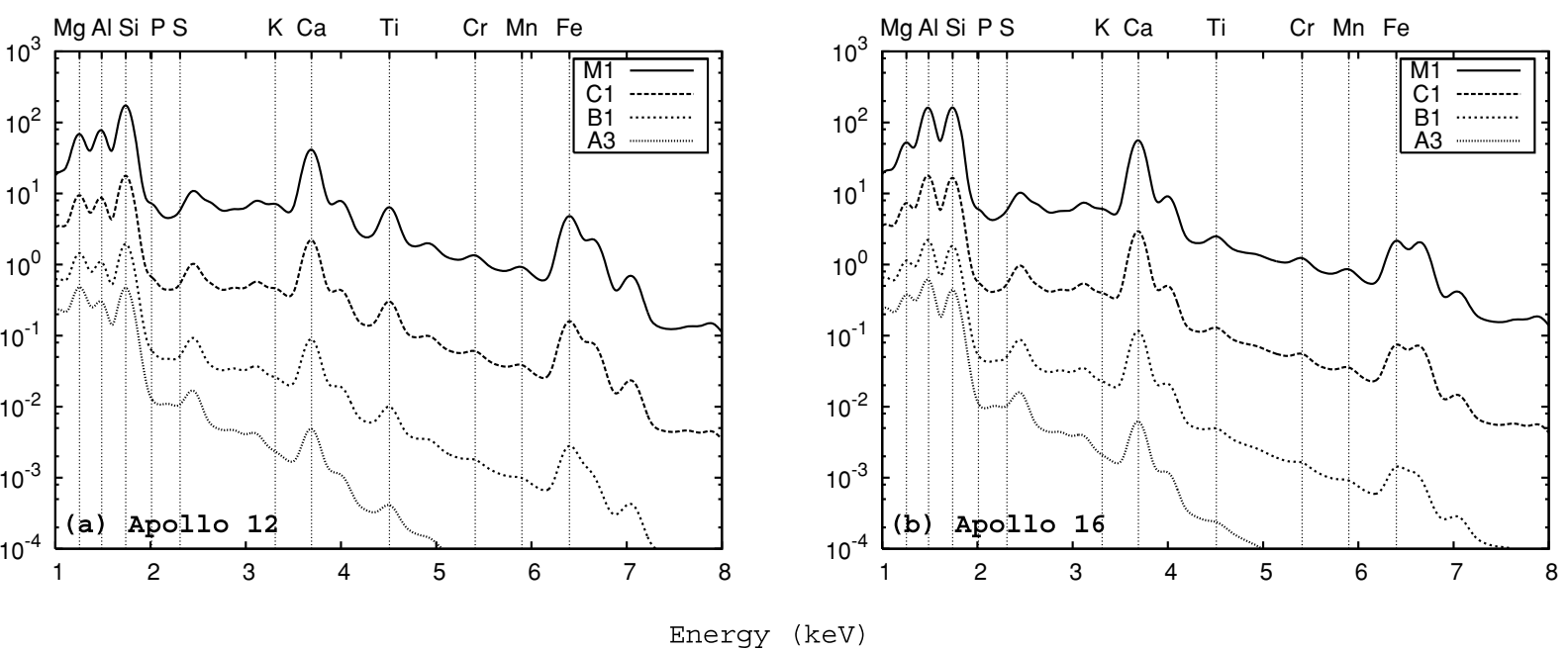

Fig. 2. Estimated lunar X-ray spectral histograms detected by the XRS onboard SELENE in the case of (a) Apollo 12 site average composition and (b) Apollo 16 site average composition. Four lines in the plots indicate spectra at the four solar activity levels shown in Fig. 1. The geometrical parameters were assumed as the solar incidence angle $\theta=0$ and the emission angle $\phi=0$.

calculated lunar spectra. Finally, in order to simulate the energy resolution of the CCDs which expands each emission line, the Gaussian distribution (normal distribution) function was applied to all the energy bins of spectra. Typical energy resolution of the CCDs experimentally estimated is approximately $150 \mathrm{eV}$ at $\mathrm{Si}-\mathrm{K} \alpha$, and $190 \mathrm{eV}$ at $\mathrm{Fe}-\mathrm{K} \alpha$ as the FWHM of the emission lines (Shirai et al., 2008).

As the results of the calculations, Figs. 2 and 3 show expected lunar X-ray spectra from the eight samples in the case of solar incidence angle $\theta=0$ (which means that solar altitude is $90^{\circ}$ ) at each solar activity level in Fig. 1. These spectral histograms include the configuration and the performance of the XRS and simulate expected X-ray counts per second. Note that photon counts in the plots are divided by $10 \mathrm{eV}$, which is the size of the energy bins of the A/D converter for XRS detectors. Energy positions of $\mathrm{K} \alpha$ lines of the major elements are also shown on the upper horizontal axes.

Figures 2 and 3 illustrate that $\mathrm{Mg}$-, Al- and $\mathrm{Si}-\mathrm{K} \alpha$ lines can be clearly seen under all conditions, whereas the higher $\mathrm{Z}$ elements $\mathrm{Ca}$, $\mathrm{Ti}$ and $\mathrm{Fe}$ lines are identifiable only under more energetic solar conditions. Probability and required integration time for these lines are discussed in Section 5. On the other hand, $\mathrm{Cr}$ and $\mathrm{Mn}$ peaks are barely identified, and $\mathrm{P}, \mathrm{S}$ and $\mathrm{K}$ are buried in the scattering backgrounds due to their small amounts of mass abundances. Detection of $\mathrm{Cr}$ and $\mathrm{Mn}$ is still possible but not probable. The other peaks in the spectra (some of them are hidden by lunar fluorescence peaks) are $\mathrm{K} \beta$ lines and scatterings of the emission lines in the solar spectra. As seen in the plots, scattering peaks of solar X-rays interfere with the lunar fluorescence peaks and produce an anomalous background in data analyses. The overall lunar scatter background shows the downwardsloping feature, which is a reflection of the solar flux.

Figure 4 shows spectra from Apollo 16 site composition by various solar incidence angles under solar levels A3 and $\mathrm{C} 1$. With decreasing solar incidence angle, the lunar scatter component becomes mild, and the fluorescent lines become more prominent. The reason for this is when the scattering angle is close to $\pi / 2$, the X-ray scattering is less effective so that the background continuum is repressed, which in contrast to the isotropic fluorescence generation. The lines of $\mathrm{Cr}$ and $\mathrm{Mn}$ in the $\mathrm{C} 1$ plot, for example, are sharper at low solar incidence angles. These results indicate that the 

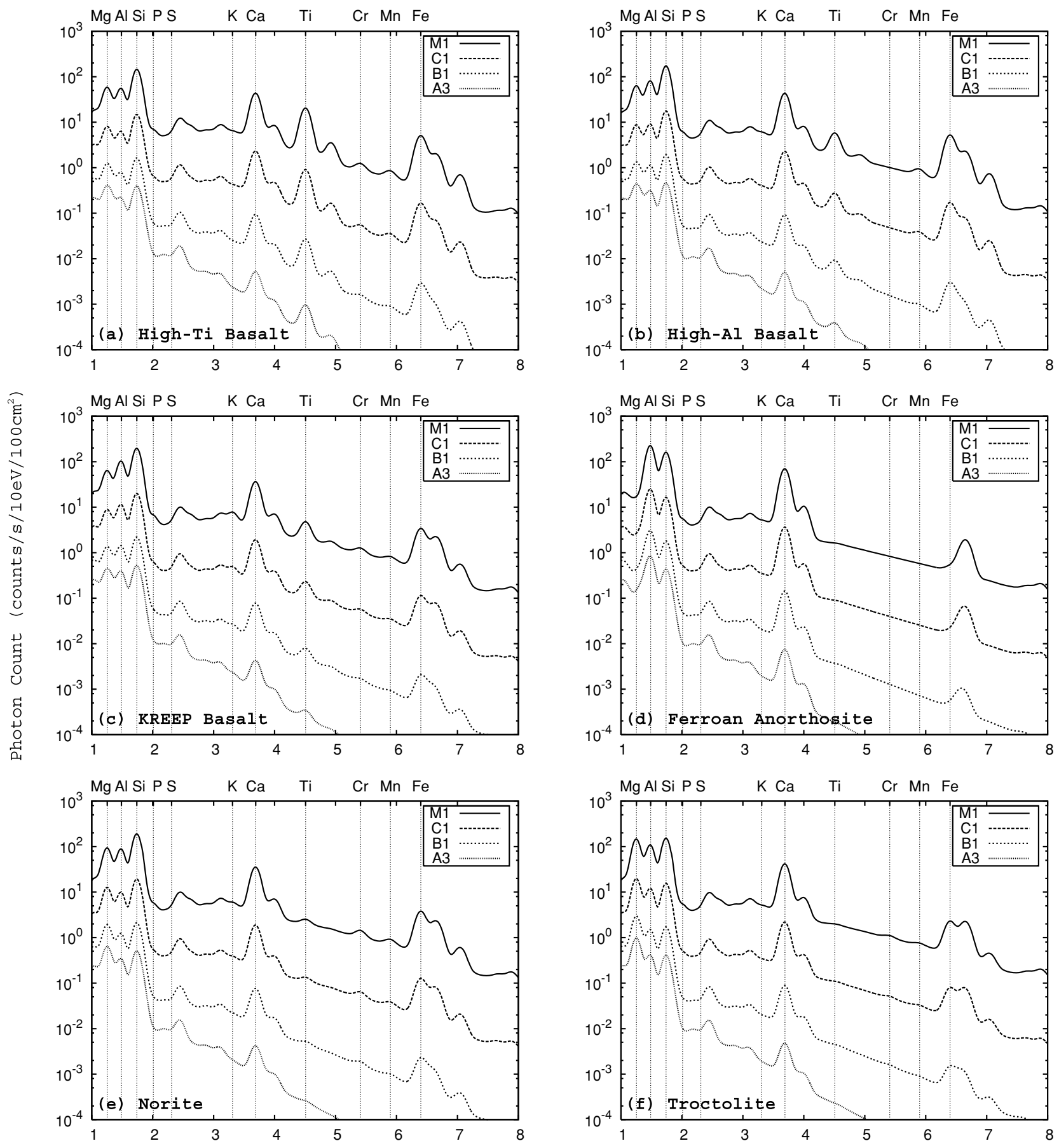

Energy $(\mathrm{keV})$

Fig. 3. Estimated lunar X-ray spectral histograms detected by the XRS are shown in the case of the six typical rocks; (a) high-Ti basalt 70215, (b) high-Al basalt 14053, (c) KREEP basalt 15382, (d) ferroan anorthosite 15415, (e) norite 77215, and (f) troctolite 76535, respectively. The assumed condition is the same as that for Fig. 2.

probability of detection of the less abundant elements will be enhanced in such a geometrical condition as when a solar flare occurs.

Relations between the normalized mass ratios of $\mathrm{Mg}, \mathrm{Al}$, $\mathrm{Ca}$, and $\mathrm{Fe}$ to $\mathrm{Si}$ and the computed $\mathrm{K} \alpha \mathrm{X}$-ray fluorescence intensity ratios to $\mathrm{Si}$ are shown in Fig. 5 for the six rock samples. Ratios of the X-ray fluorescence intensity from the same sample significantly vary with the change in solar activity. Fractions of fluorescent intensity of $\mathrm{Mg}$ and $\mathrm{Al}$, which form lower energy lines than $\mathrm{Si}$, decrease under the condition of an intense Sun as contrasted to $\mathrm{Ca}$ and $\mathrm{Fe}$ (and also Ti). This is because the spectral profile of active Sun efficiently excites heavier elements.

As mentioned in Section 3, the relation between mass ratio and fluorescence photon count ratio, both normalized to $\mathrm{Si}$, is theoretically nonlinear. To determine the elemental abundance ratio, linear working curve analyses are often available for small quantity fluctuations between similar total compositions. However, for accurate composition analyses, it is necessary to compare the observed data with the 


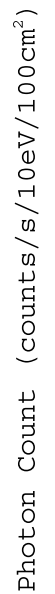
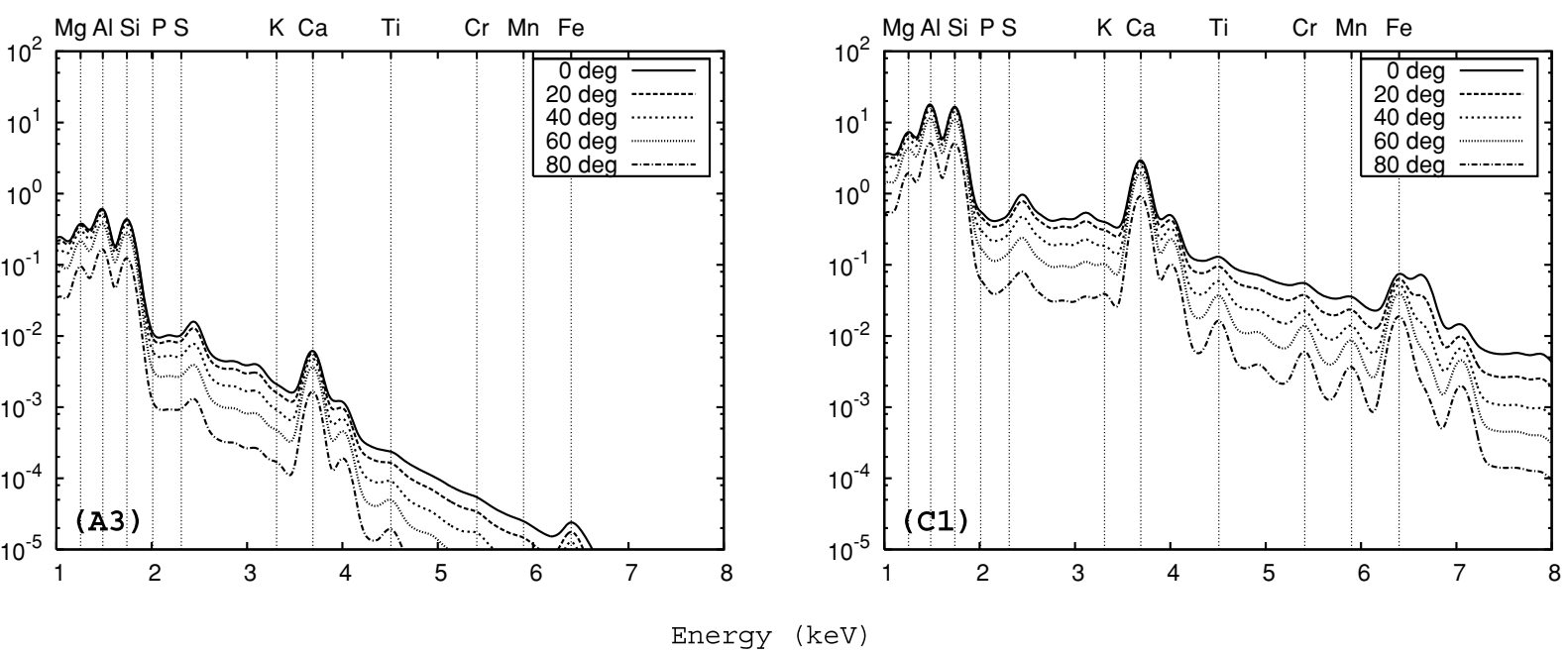

Fig. 4. Solar incidence angle dependency of the lunar X-ray spectra at two solar activity levels of A3 and C1. Elemental composition of the sample was assumed to be the Apollo 16 site average composition representing highland soil.
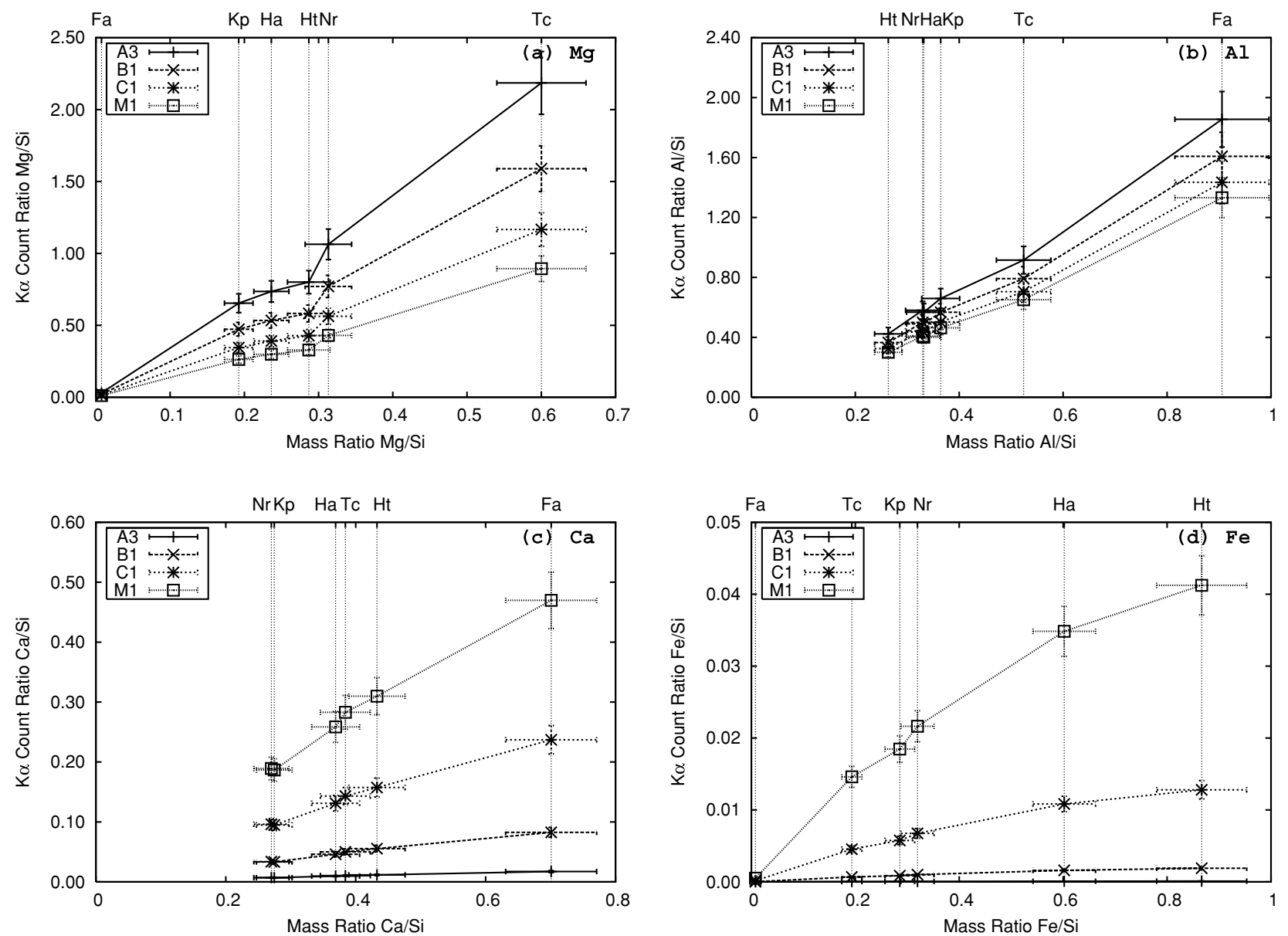

Fig. 5. Mass ratio to $\mathrm{Si}$ in the samples vs. computed $\mathrm{K} \alpha$ photon count ratio to $\mathrm{Si}$ are plotted for $\mathrm{Mg}, \mathrm{Al}, \mathrm{Ca}$ and Fe. The keys on the upper horizontal axes are (Ht) high-Ti basalt 70215, (Ha) high-Al basalt 14053, (Kp) KREEP basalt 15382, (Fa) ferroan anorthosite 15415, (Nr) norite 77215 and (Tc) troctolite 76535, respectively. The four lines in the figures indicate the solar activity levels of A3, B1, C1, and M1. The error bars of the plots show $\pm 10 \%$ of the ratios.

theoretical model, including the nonlinear matrix effect by major components.

The results in Fig. 5 show that the XRS can distinguish the six typical rock types in Table 3 , if the line intensity ratios of the target elements to $\mathrm{Si}$ are measured within
$10 \%$ accuracy. The rocks representing highland, ferroan anorthosite, norite and troctolite can be distinguished by the ratio of $\mathrm{Mg} / \mathrm{Si}$ and $\mathrm{Al} / \mathrm{Si}$ which are the most sensitive signals for the XRS. Additional information of $\mathrm{Ca}$, Ti and Fe facilitate a more accurate identification, especially in the 

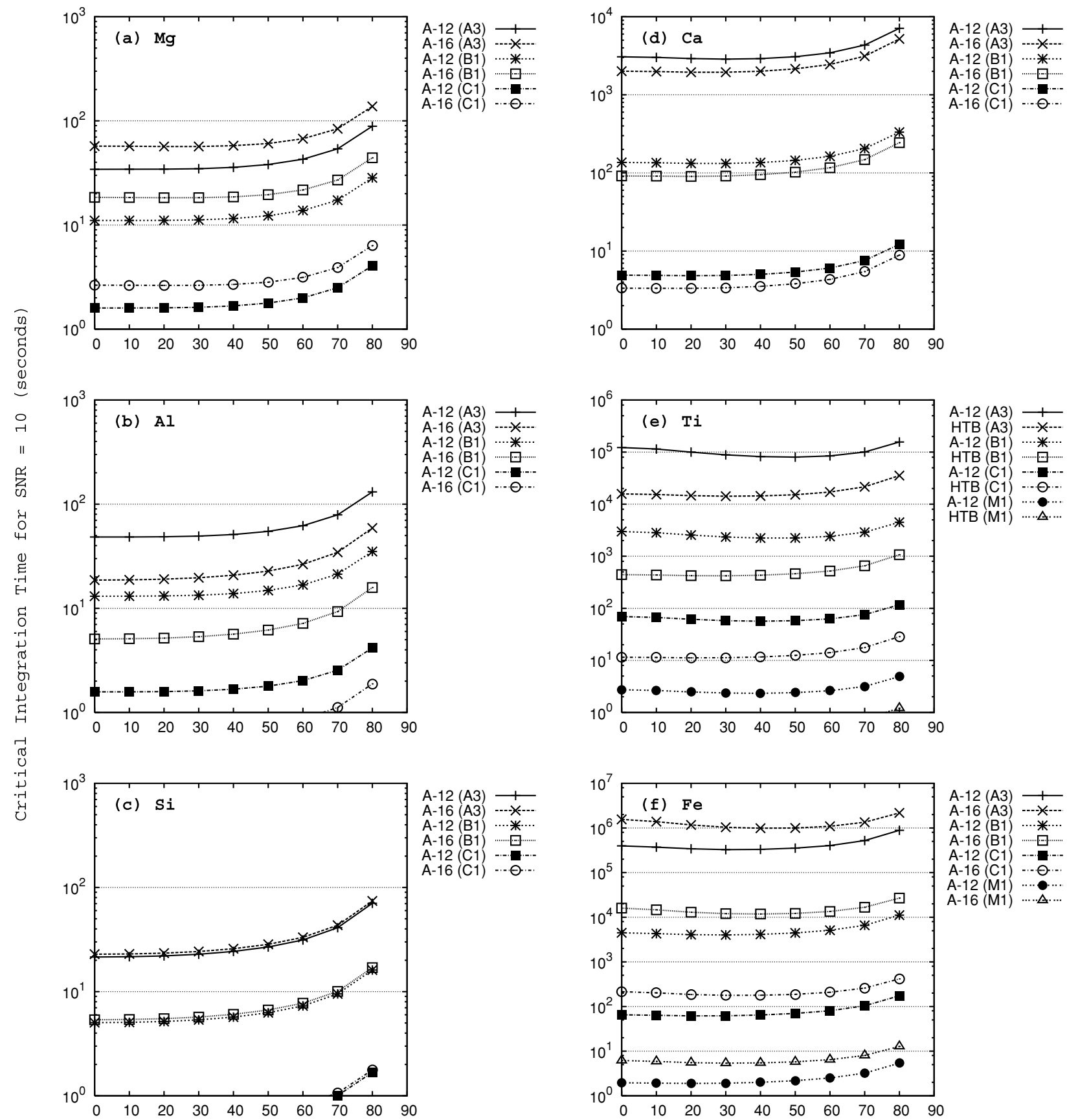

Solar Incidence Angle (degrees)

Fig. 6. Plots of required measuring time (seconds) to achieve the critical sensitivity ( $\mathrm{SNR}=10$ ) are shown for $\mathrm{Mg}, \mathrm{Al}, \mathrm{Si}, \mathrm{Ca}$, $\mathrm{Ti}$ and $\mathrm{Fe}$ for solar altitudes from $10^{\circ}$ to $90^{\circ}$. The explanatory notes in the figures mean (A-12) Apollo 12 site average composition representing lunar maria, (A-16) Apollo 16 site average representing highland and (HTB) high-Ti mare basalt 70215, under each solar level of A3, B1, C1, and M1, respectively. Note that Ti was plotted for the compositions of Apollo 12 site average (A-12) and high-Ti basalt (HTB) listed in Table 3.

mare basalts which are characterized by ratios of heavier elements.

\section{Analysis of Critical Integration Time}

The integration times required for detecting the target elements of $\mathrm{Mg}, \mathrm{Al}, \mathrm{Si}, \mathrm{Ca}, \mathrm{Ti}$ and $\mathrm{Fe}$ and their subsequent along-the-track spatial resolutions are discussed in this section. The aim of the XRS is to map the elemental abundance ratios (ratios to $\mathrm{Si}$ ) on the lunar surface within $10 \%$ error. We also investigated the critical integration times to fulfill higher than 10 of signal-to-noise ratio (SNR) of the X-ray fluorescence peak. Here, we defined the SNR of the peaks by the following formula,

$$
\mathrm{SNR} \equiv \frac{S}{\sqrt{S+B}}
$$

where $S$ is the count of the fluorescent X-rays integrated over energy range of $\pm 1 \sigma$ from the peak center ( $\sigma$ is related to the FWHM according to $\sigma=\mathrm{FWHM} /(2 \sqrt{2 \ln 2})$, about $68 \%$ of fluorescent $\mathrm{X}$-rays are contained in $\pm 1 \sigma$.), and $B$ is the count of the background (scattering) photons 
integrated over the same range of $S$. The critical integration times for $\mathrm{K} \alpha$ lines of the six target elements to achieve $\mathrm{SNR}=10$ were calculated individually for the samples in Apollo 12 and the 16 site average soil compositions in Table 3. Four levels of solar activity and solar incidence angle increments of ten degrees were assumed. It is noted that the SNR obtained here represents an ideal condition of the detector, and Eq. (13) does not include instrumental noise or other background sources.

The results of $\mathrm{Mg}, \mathrm{Al}$ and $\mathrm{Si}$ are plotted in Fig. 6(a-c). Compositional ratios of these elements are the most fundamental information to identify dominant rock types and the degree of lunar geological processing. It seems that abundances of these elements are detectable within $60 \mathrm{~s}$ of integration in most cases of solar quiet A3, while Si is easier, and can be detected in less than $30 \mathrm{~s}$. If strong solar flares over $\mathrm{C} 1$ occur, detection can be made within a few seconds. Since SELENE passes at a ground speed of $1.5 \mathrm{~km} / \mathrm{s}$, this integration time means that elemental mapping of the three major elements is simply conducted by spatial resolution along the track of $<90 \mathrm{~km}$ and $20 \mathrm{~km}$ under the normal and active Sun conditions, respectively.

On the other hand, Fig. $6(\mathrm{~d}-\mathrm{f})$ shows that identification of the heavier elements $\mathrm{Ca}, \mathrm{Ti}$ and $\mathrm{Fe}$ require longer time integration by a factor of $>10^{3}$ during solar quiescence. Since the estimated spatial resolutions of these sub-major elements are more than $4500 \mathrm{~km}$ for a quiescent Sun, this is almost lunar hemispheric or bulk average analysis. Therefore, strong solar flares are necessary for spatially focused elemental composition analyses of these elements. For the spatial resolution of $20 \mathrm{~km}$ along the track, $\mathrm{C} 1$ or stronger flares are required for $\mathrm{Ca}$, and $\mathrm{M} 1$ or stronger for $\mathrm{Ti}$ and $\mathrm{Fe}$.

The remarkable thing is that the critical integration times at the high solar incidence angle (close to $80^{\circ}$ ) are only at maximum two or three times as long as those at the low incidence angle (close to $0^{\circ}$ ). Elemental mapping of the polar regions $\left(60^{\circ}\right.$ to $80^{\circ}$ latitude) or the case of high orbital $\beta$ angle observations during dawn and dusk time was often considered to be ineffective, but practical observations can possibly be carried out with a little longer integration time.

\section{Concluding Remarks}

In this study, we have computed simulations for the lunar X-ray spectra observed by the XRS onboard SELENE with the improved X-ray interaction model and the solar $\mathrm{X}$-ray spectral data, including emission lines from solar elements. The critical measuring times and the subsequent along-the-track spatial resolutions for the pulse height analyses of the target elements $(\mathrm{Mg}, \mathrm{Al}, \mathrm{Si}, \mathrm{Ca}, \mathrm{Ti}$ and $\mathrm{Fe})$ were also estimated, which is applicable to the actual operations of SELENE. Anticipated along-the-track spatial resolution with $\mathrm{SNR}=10$ for $\mathrm{Mg}, \mathrm{Al}$ and $\mathrm{Si}$ is $<90 \mathrm{~km}$, and $20 \mathrm{~km}$ during higher solar activity. With these resolutions, the distribution of dominant rock types in the lunar maria, highlands or large craters of the whole lunar surface can be mapped in detail.

The spatial resolution is correlated with the field-of-view of the XRS. With a double-wide footprint, for instance, the integration time and the along-the-track spatial resolution become half, whereas the swath of the measurements is broadened. The current configuration of the footprint size was optimized principally for the lunar X-ray flux and the corresponding machine power of data processing, nevertheless, the best spatial resolution of $20 \times 20 \mathrm{~km}^{2}$ at active solar conditions is comparable to the central peaks of large craters, such as Tsiolkovsky crater. The central peak is one of the most interesting areas for studies of lunar crustal processes (e.g., Tompkins and Pieters, 1999; Wieczorek and Zuber, 2001), and this is one criterion for configuring the field-of-view. The XRS is potentially sensitive to resolve $\mathrm{Mg} / \mathrm{Si}$ and $\mathrm{Al} / \mathrm{Si}$ in the scale of central peaks, ejecta of large impact basins or valley-like features. Mapping of $\mathrm{Ca}$, $\mathrm{Ti}$ and $\mathrm{Fe}$ will be usually done by relatively rough resolution that significantly depends on the solar activity level, but point abundance information within $100 \mathrm{~km}$ or smaller spatial resolution can be obtained during intensive flares. In any case, analyses of large geological features can be performed for the three elements during the 1-year long observation period, since a few intensive solar flares are likely to occur while the orbiter observes a part of the large area.

The spatial resolutions discussed above are those along the track of a single path. Spatial resolution is, however, practically improved with adequate data analyses and using multiple path data that covers the same area. On the other hand, instrumental noise or other background sources were not included in the calculations. It is generally known that $\mathrm{X}$-ray CCDs suffer from radiation damage, which appears as increasing continuum background of spectra and degradation of the energy resolution, which in turn arise from exposure to energetic particles during passage through the Earth's radiation belts and a long time stay in space. Sufficient spectral analyses to remove the effects due to these phenomena should therefore be performed.

The relation between the fluorescence intensity ratio and elemental mass ratio varies with the change in solar activity so that a fixed calibration curve is not available in data analyses. In addition, lunar X-ray spectra include scattered $\mathrm{X}$-rays of solar X-ray line spectra such as ionized S, Ar or Fe with the peaks at $2.5,3.1$, or $6.7 \mathrm{keV}$, respectively. These scattered lines should be carefully accounted for in the lunar fluorescence peak height analyses. To avoid these problems, concurrent monitoring of solar X-rays during the actual lunar operations and investigations of detailed solar spectra are needed. In the actual XRS operations, the SOLB and SOL-C provide the solar X-ray profile at the time, and the solar spectral data can be deconvolved in detail by fitting to solar X-ray models, such as MEKAL model.

The lunar X-ray model used in this study assumes a flat homogeneous lunar surface, but macro-scale topographic features, such as slopes of the surface, and micro-scale roughness by particle size distribution of the lunar soil affect more than $10 \%$ of photon counts of both fluorescence and scatterings especially at large phase angle (Maruyama et al., 2007, 2008). These effects must be considered for more accurate modeling of lunar X-ray spectra with experimental results or numerical simulations. Modeling photon interactions by the Monte-Carlo technique would be one possible investigation method. Experimental investigations of the effects will be also conducted by the observations from the XRS onboard SELENE. 
Acknowledgments. The authors acknowledge detailed comments and support by Dr. Yukio Yamamoto, Dr. Hiroaki Shiraishi, and Mr. Takehiko Arai in the XRS team. The authors thank two anonymous reviewers for helpful comments that improved this paper.

\section{References}

Adler, I. and J. I. Trombka, Orbital chemistry-Lunar surface analysis from the X-ray and gamma ray remote sensing experiments, Phys. Chem. Earth, 10, 17-43, 1977.

Adler, I., J. I. Trombka, J. Gerard, P. Lowman, R. Lamothe, R. Schmadebeck, H. Blodget, E. Eller, L. Yin, R. Lamothe, P. Gorenstein, and P. Bjorkholm, Apollo 15 geochemical X-ray fluorescence experiment: Preliminary report, Science, 175, 436-440, 1972a.

Adler, I., J. I. Trombka, J. Gerard, P. Lowman, R. Schmadebeck, H. Blodget, E. Eller, L. Yin, R. Lamothe, G. Osswald, P. Gorenstein, P. Bjorkholm, H. Gursky, and B. Harris, Apollo 16 geochemical X-ray fluorescence experiment: Preliminary Report, Science, 177, 256-259, $1972 b$.

Adler, I., J. I. Trombka, L. I. Yin, P. Gorenstein, P. Bjorkholm, and J. Gerard, Lunar Composition from Apollo Orbital Measurements, Naturwissenschaften, 60, 231-242, 1973a.

Adler, I., J. I. Trombka, R. Schmadebeck, P. Lowman, H. Blodget, L. Yin, and E. Eller, Results of the Apollo 15 and 16 X-ray experiment, Proc. Lunar Sci. Conf., 4, 2783-2791, 1973b.

Bearden, J. A., X-ray wavelengths, Rev. Mod. Phys., 39, 78-124, 1967.

Brusa, D., G. Stutz, J. A. Riveros, J. M. Fernández-Varea, and F. Salvat, Fast sampling algorithm for the simulation of photon Compton scattering, Nucl. Instrum. Methods Phys. Res. A, 379, 167-175, 1996.

Chantler, C. T., Detailed tabulation of atomic form factors, photoelectric absorption and scattering cross section, and mass attenuation coefficients in the vicinity of absorption edges in the soft $X$-ray $(Z=30-36$, $\mathrm{Z}=60-89, \mathrm{E}=0.1 \mathrm{keV}-10 \mathrm{keV})$, addressing convergence issues of earlier work, J. Phys. Chem. Ref. Data, 29, 597-1056, 2000.

Chantler, C. T., K. Olsen, R. A. Dragoset, J. Chang, A. R. Kishore, S. A. Kotochigova, and D. S. Zucker, X-ray form factor, attenuation and scattering tables (version 2.1), [Online] Available: http://physics.nist.gov/ffast [2007, March], National Institute of Standards and Technology, Gaithersburg, MD, 2005.

Clark, P. E., X-ray spectrometry for remote exploration of mercury and the Moon, Adv. Space Res., 19, 1539-1549, 1997.

Clark, P. E. and J. I. Trombka, Remote X-ray spectrometry for NEAR and future missions: Modeling and analyzing X-ray production from source to surface, J. Geophys. Res., 102(E7), 16,361-16,384, 1997.

Cromer, D. T. and J. T. Waber, Atomic scattering factors for X-rays, in International Tables for X-Ray Crystallography (Vol. 4), edited by J. A. Ibers and W. C. Hamilton, The Kynoch Press, Birmingham, 1974.

Grande, M., R. Browning, N. Waltham, D. Parker, S. K. Dunkin, B. Kent, B. Kellett, C. H. Perry, B. Swinyard, A. Perry, J. Feraday, C. Howe, G. McBride, K. Phillips, J. Huovelin, P. Muhli, P. J. Hakala, O. Vilhu, J. Laukkanen, N. Thomas, D. Hughes, H. Alleyne, M. Grady, R. Lundin, S. Barabash, D. Baker, P. E. Clark, C. D. Murray, J. Guest, I. Casanova, L. C. D’Uston, S. Maurice, B. Foing, D. J. Heather, V. Fernandes, K. Muinonen, S. S. Russell, A. Christou, C. Owen, P. Charles, H. Koskinen, M. Kato, K. Sipila, S. Nenonen, M. Holmstrom, N. Bhandari, R. Elphic, and D. Lawrence, The D-CIXS X-ray mapping spectrometer on SMART-1, Planet. Space Sci., 51, 427-433, 2003.

Grande, M., B. J. Kellett, C. Howe, C. H. Perry, B. Swinyard, S. Dunkin, J. Huovelin, L. Alha, L. C. D'Uston, S. Maurice, O. Gasnault, S. Couturier-Doux, S. Barabash, K. H. Joy, I. A. Crawford, D. Lawrence, V. Fernandes, I. Casanova, M. Wieczorek, N. Thomas, U. Mall, B. Fo- ing, D. Hughes, H. Alleyne, S. Russell, M. Grady, R. Lundin, D. Baker, C. D. Murray, J. Guest, and A. Christou, The D-CIXS X-ray spectrometer on the SMART-1 mission to the Moon-First results, Planet. Space Sci., 55, 494-502, 2007.

Hayakawa, S. and M. Matsuoka, Characteristic X-rays from the lunar surface, Report. Ionosphere and Space Res. in Japan, 16, 341-357, 1962.

Hubbell, J. H., P. N. Trehan, N. Singh, B. Chand, D. Mehta, M. L. Garg, R. R. Garg, S. Singh, and S. Puri, A review, bibliography, and tabulation of $\mathrm{K}, \mathrm{L}$, and higher atomic shell X-ray fluorescence yields, J. Phys. Chem. Ref. Data, 23, 339-364, 1994.

Kato, M., S. Sasaki, K. Tanaka, Y. Iijima, and Y. Takizawa, The japanese lunar mission SELENE: Science goals and present status, Adv. Space Res., 2007 (in press).

Kuwada, Y., T. Okada, and H. Mizutani, Particle size effect in X-ray fluorescence, Proc. ISAS Lunar Planet. Symp., edited by H. Mizutani and M. Kato, ISAS, Sagamihara, Japan, 30, 212-215, 1997.

Maruyama, Y., K. Ogawa, T. Okada, and M. Kato, Particle size effect in $\mathrm{X}$-ray fluorescence and its implication to planetary XRF spectroscopy, Proc. Lunar Planet. Sci. Conf., XXXVIII, \#1186, 2007.

Maruyama, Y., K. Ogawa, T. Okada, and M. Kato, Laboratory experiments of particle size effect in X-ray fluorescence and implications to remote $\mathrm{X}$-ray spectrometry of lunar regolith surface, Earth Planets Space, 60, this issue, 293-297, 2008.

McKay, D. S., G. Heiken, A. Basu, G. Blanford, S. Simon, R. Reedy, B. M. French, and J. Papike, The lunar regolith, in Lunar Sourcebook, edited by G. H. Heiken, D. T. Vaniman, and B. M. French, Cambridge University Press, 1991.

Okada, T., Basic study for XRF spectrometry of planetary surfaces, $\mathrm{PhD}$ thesis, University of Tokyo, Japan, 1996 (in Japanese).

Okada, T., M. Kato, Y. Yamashita, K. Shirai, Y. Yamamoto, T. Matsuda, H. Tsunemi, and S. Kitamoto, Lunar X-ray spectrometer experiment on the SELENE mission, Adv. Space Res., 30, 1909-1914, 2002.

Ribberfors, R. and K. F. Berggren, Incoherent-X-ray-scattering function and cross section $\left(\mathrm{d} \sigma / \mathrm{d} \Omega^{\prime}\right)_{\text {incoh }}$ by means of a pocket calculator, Phys. Rev. A, 26, 3325-3333, 1982.

Salem, S. I., S. L. Panossian, and R. A. Krause, Experimental K and L relative X-ray emission rates, Atom. Data Nucl. Data Tables, 14, 91109, 1974.

Shirai, K., T. Okada, Y. Yamamoto, T. Arai, K. Ogawa, H. Shiraishi, M Iwasaki, M. Arakawa, M. Grande, and M. Kato, Instrumentation and performance evaluation of the XRS on SELENE orbiter, Earth Planets Space, 60, this issue, 277-281, 2008.

Shiraiwa, T. and N. Fujino, Theoretical calculation of fluorescent X-ray intensities in fluorescent $\mathrm{X}$-ray spectrochemical analysis, Japanese $J$. Appl. Phys., 5, 886-899, 1966.

Taylor, G. J., P. Warren, G. Ryder, J. Delano, C. Pieters, and G. Lofgren, Lunar rocks, in Lunar Sourcebook, edited by G. H. Heiken, D. T. Vaniman, and B. M. French, Cambridge University Press, 1991.

Tompkins, S. and C. M. Pieters, Mineralogy of the lunar crust: Results from Clementine, Meteorit. \& Planet. Sci., 34, 25-41, 1999.

Wieczorek, M. A. and M. T. Zuber, The composition and origin of the lunar crust: Constraints from central peaks and crustal thickness modeling, Geophys. Res. Lett., 28, 4023-4026, 2001.

Yamamoto, Y., T. Okada, H. Shiraishi, K. Shirai, T. Arai, K. Ogawa, K. Hosono, M. Arakawa, and M. Kato, Current status of X-ray spectrometer development in the SELENE project, Adv. Space Res., 2007 (in press).

K. Ogawa (e-mail: ogawa@planeta.sci.isas.jaxa.jp), T. Okada, K. Shirai, and M. Kato 